\title{
Ueber Flockung von Kolloiden durch Nichlleiter.
}

\author{
Von Paul Klein.
}

(Eirngegangen ana 27. Juli 1921.)

(Aus der Technischen Huchschule Chariottenburg.)

Eine Anzahl von Einzelergebnissen über Flockungen durch indifferente Nichtleiter bat man, um dieselben mit der herrschenden Theorie in Einklang zu bringen, mehrfach aut do gieichzeitige Gegenwart von Elektrolyten zurickzuführen gesucht (s. w. u. H. Freundlich und P. Rona sowie O. Meyerhoff). Die im folgenden wiedergegebene Arbeit möge zeigen, bis zu welchem Grade dieser Schlub zulässig ist.

K. Spirol) hat die Flockung von Eiweisstoffen durch Alkohole quantilativ verfolgt und gezeigt, dab Eiereiweib durch z.B. zwei- bis vierprozentigen Amylalkohol gellockt werden kann. Moore und Roaf ${ }^{2}$ zeigten $u$. a., dab auch Chloroform eine derartige Flockung verursacht. R a m s de $n^{3}$ ) wies darauf hin, dab Chloroform, Aethyläther, i-Amylalkohol und Schwefelkohlenstoff manche Kolloide fallen.

Batelli und Stern ${ }^{4}$ ) stellen fest, dab Nukleoproteide durch eine gröBere Anzahl oberflächenaktiver Stoffe geflockt werden. Warburg und Wies $1^{5}$ ) fanden, daB Hefeprebsaft durch verschiedene Anästhetika gefällt wird.

Endlich erwähnen Bechhold, Dede und Reine $t^{0}$ ) in einer allerdings nach Fertigsteliung dieser Arbeit veroffentlichien Mitteilung, dal man Hämoglobin and Eiweißlösungen, ferner Suspensionen fester Stoffe, wie Ton usw. durch Schütteln mit Benzol flocken könne.

Bekannt sind ferner die Sensibilisations versuche von Kruyt und Duin sowie Freundlich und Rona ${ }^{7}$ ). Diese Autoren zeigten, dab bei Gegenwart oberflächenaktiver Nichtleiter in einem Sole von Eisenoxydhydrat Flockungen durch weitaus geringere Mengen von Elektrolyten herbeigeführt werden. als ohne deren Gegenwart. Die Elektrolyten werden durch die Nichtleiter "sensibilisiert", nach der Meinung Freundlich's und Rona's dadurch, dab die Dielelektritätskonstante des Lösungsmittels durch

1) K. Spiro, Beitr. z. chem. Physiol, u. Pathol. 4, 300 (1903).

2) G. Moore u. H. E. Róaf, Proc. Roy. Soc. Lond. [B.] 77, 86 (1906).

9) C. E. Ramsden, Zeitschr. f. phys. Chem. 2, 47.

4) F. Batelli u. L. Stern, Biochem. Zeitschr. 52, 226 (1912).

b) O.Warburg u. F. Wlese1, Pliuger's Arch. $t$. d. ges. Physiol. 144, 465 (1912).

6) H.Bechhold, L.. Dede u. L, Reiner, Koll. Zeitschr. 28, 6 (1921).

7) H. Freundlich u. P. Rona, Biochem. Zeitschr. 81, 87 (1917). die Nichtleiter vermindert wird 7 . Freundich und $R$ on a sprachen nun die Ansichr aus, daf es sich bei den Versuchen von $B$ atelli und Stern vermutlich atach nur um Sensibilisationen handle und 0 . Me yer h of ${ }^{9}$ ) ist geneigt, ganz allgemein anzunehmen, dab auch bei den Versuchen anderer Autoren es sich wohl nur um Sensibilisation gehandelt habe, da ja Eiweiblosungen itn allgemeinen nicht als elektrolytfrei anzusehen sind.

In der Tat hat ja diese Ansicht eine gewisse Berechtigung, indessen die folgenden Versuchsreihen werden doch zeigen, da ganz $z$ weifel. los a uch Kolloide ohne Gegenwart ron Elektrolyten durch indifierente Nichtleiter geflockt werden.

Herr Professor. J.Tra ube, in dessen Laboratorium die vorliegende Arbeit ausgeführt wurde, gab mir Kenntnis von gewissen Versuchsreihen, welche von ihm nicht veröffentlicht worden sind und welche sich auf die Flockung von Kohle und Schwelelsuspensionen durch oberflächenaktive Nichtleiter, wie i-Amylalkohol, tertiärer Amylalkohol, Heplylalkohol, Kaprylsäure, i-Amylazetat usw, beziehen. Professor Traube fand, dak eine Flockung dann eintrat, wenn die wässerige Lösung an Amylaikohol usw. gesättigt war. Amylalkohol and Butylalkohol wirkten flockend, dagegen nicht Propylallohol und Aethylalkohol, weil diese Stoffe sich in jedem Verhältnis in Wasser losten.

Versuche mit suspendierten Pulvern.

Nach dieser Richtung lagen schon eine Anzabl von Versuchen vor, namentich von F. B. Hof mann ${ }^{10}$ ). Die erwähnte Arbeit von Bechhold, Ded e and Reiner erschien erst, nachdem meine Versuche bereits ausgeführt waren.

Es wurden von mir wässerige Suspensionen untersucht von Kalziumsulfat (Anhydrit), Bariumsulfat, Bleisulfat, Mangansuperoxyd, Quecksilberjodid, Schwefelblumen, kolloidem Schwefel,

8) Diese Theorie beruht auf einer entsprechenden Angabe von Wo. Ostwald, der u. a. auch eine eigene Beobachtung, nach welcher ein Silberhydrosol durch Sätigen mit Propylalkohol gefalt wird duch obigen Umstand erklart (s. Grundrib d. Kolloidchem. I, Aufl. Dresden 1909, S. 471).

9) 0 . Meyerhoff, Pflüger's Arch. f. d. ges. Physiol. 86, 325 (1918).

10) F.B. Ho fm a n n, Zeitschr. f. physik. Chem. 83, 385 (1913); vgl. weitere Literatur bei $\mathrm{H}$. B e ch hold, L. Dede ti. L. Reiner, loc, cit. 
Kupfer, Silber, Zink, Eisen und Kohle. Als zweckmäßig erwies es sich, die Flockungstnittei in Form der äthylalkoholischen Lösung einzufuhren, da aũ diesem Wege eine wesentlich feinere Verteilung herbeigeführt wurde als durch Schütreln. Als Flockungsmittel wurden verwand: i A Amylakohol, Heptylakohol, Chloroform, Tetrachlorkohlenstoff, Benzol, Anilin, Diphenylamin. Kapronsänre, Heptylsäure. Kaprylsăure ind Nonylsäure.

In Uebereinstimmung mit anderen Beobachtungen wurde festgestellt, das in allen Fällen die festen Teilchen an der AuBenflache der Kügeichen des Flockungsmittels adsorbiert wurden. Die kleinen mit fester Substanz mehr oder weniger beladenen Kügelchen vereinten sich nun teilweise zu Flöckchen und Flocken und je nach ihrem spezifischen Gewichte stisgen dieselben an die Oberfläche empor oder sanken zu Boden oder hielten sich längere Zeit schwebend. Chloroformtröpfchen senkten sich zu Boden, Benzoltropfchen, wenn nicht zu schwer beladen, begaben sich an die Oberfläche und bei passendem Mischungsverhaltnis von Chloroform und Benzol konnte man die Troptchen beliebig lange Zeit schwebend erhalten. Wern auch bei den Flotationsvorgängen bei der Erzgewinnung die Mengenverhäitnisse andere sind, so haben diese Versuche doch auch nach dieser Richtung einiges'lateresse.

Versuche mit einem Arsensulfidsol.

Das Sol, welches $9 \mathrm{Millimol}$ Arsensulfid im Liter enthielt, wurde nach FreundIich durch Einleiten von Schwefelwasserstof in eine Lösung von arseniger Saure und nachherigern Vertreben des therschussigen Schwefelwasserstoffs durch Wasserstoff hergestellt. Das Sol Katin als elektrolytfrei angesehen werden.

$Z_{u}$ je 3 cum dieses Sols wurde je $1 \mathrm{~cm}$ einer äthylalkoholischen Losung der oben genannten oberflächenaktiven Nichtleiter von verschiedener Konzentration zugegeben und es wurde festgestellt, dab siets dann, wenn die wasserige Losung an dem Nichtleiter übersätigt war, eine irreversible Flockung von Arsensulfid ein" trat. Hier handelt es sich um keine Sensibilisation. Versuche, durch Zusatz der Nichtleiter allein Flockung herbeizufïiren, scheiterten. Dagegen trat bei Zusatz der alkoholischen Lösung dieser Stoffe augenblicklich eine Flockung ein. Bemerkenswert waren die Optima der Wirkungen für gewisse mittlere Kon- zentrationen. Die anbel befindliche Photographie (Fig. 1) stellt eine Reihe von Versuchen dar,

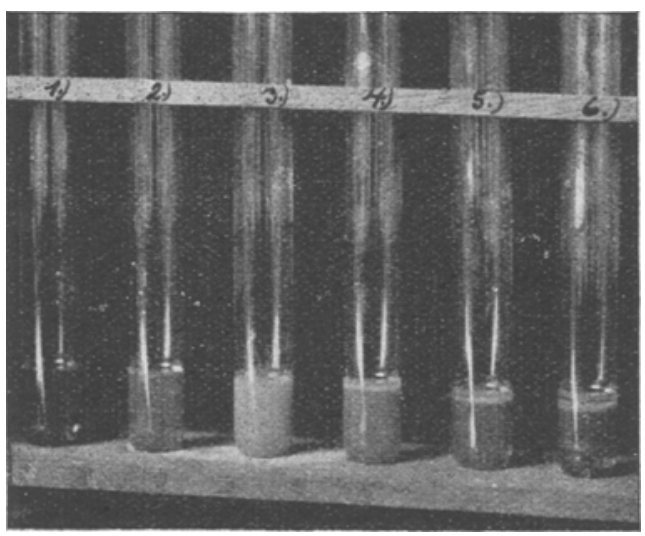

Fig. 1

bei denen zu je $3 \mathrm{ccm}$ Arsensulfidsol der Reihe nach je 1 ccm einer $10-, 16-, 20-, 25-, 30$ - bzw. 40 prozentigen athylakoholischen Lösung von i Amylalkohol zugesetzi wurde. Das Flockungsund Emulgierungsoptimum zeigt Rohre 3; es entspricht einer Konzentration von etwa 5 Proz. i-Amylalkohol auf die gesamte Funssigkeitskeitsnenge berechnet. Indessen die Flockung beginnt bereits in Rohre 2 , in welcher die Konzertration von 4 Proz ciner etwa gesättigten i-Amylalkohollösung entspricht. Aehnliche Optima der. Flockung wurden auch in anderen Fälien beobachtet. So war beispielsweise die Flockung bei Zusatz von 1 ccm einer einprozentigen Heptylsüzure- bzw. Nonylsäurelösung zu $3 \mathrm{ccm}$ des Arsensulfidsols weit stärker als bei Zusaz von $1 \mathrm{ccm}$ einer zehnprozentigen Losung. 1 ccm einer 25 prozentigen Anilinlösung zu $3 \mathrm{ccm}$ Arsensulfidsol flockte stärker als die gleiche Menge einer 50 prozentigen Loosung usw. Mit allen den oben genannten oberflachenaktiven Stoffen wurden Flockungen beobachtet. Für die Ansammlung der Flocken am Boden oder an der Oberfläche des GefăBes war auch hier naturgenab das spezifische Gewicht entscheidend.

\section{Versuche mit Albuninlösung.}

$3 \mathrm{ccm}$ einer einprozentigen Eieralbuminlösung (von Kahibaum) wurden gleichfalls mit je $1 \mathrm{ccm}$ einer äthylahoholischen Losung obiger oberflächenaktiver Stofe in wechselnden Konzentrationen versetzt. Es wurde in allen Fällen, sobald die Lösungen abersättigt wurden, eine irreversible Flockung erziett. Oberllächen. aktive Stoffe, welche in jedem Verhältnis in 
Wasser löslich sind, ergaben keine Plockung, wenn die Konzentrationen nirht allzu grob sind Dieser Umstaud allein zegt schon, dub es sich hiser nicht um einen Sersibilisierungsvorgang handelt.

Sehr bemerkenswert ist namentlich auch von Standpunkte von Tr a u b e's Narkosetheorie aus die Irreversibiliất der Eiweibfiockung. $\mathrm{Da}$ die flockenden Narkotika sich an der Zellgrenz fläche konzentrieren, so führen sie hier irreversible Eiweisflockungen herbei, welche für die schädliche und unter Umständen tödliche Wir. kung der Narkotika die hauptsächliche Ursache sein dürften.

Versuche mit elektronegativ geladenen Solen von Gold, Kieselsäure, Eisenhydroxydund $Q$ uecksilbersulfid.

Es gelang mir, nahezu elektrolytfreie rote Goldsole, weiche durch Einleiten vor reinem Kohlenoxyd in eine verdünnte neistrale Lösung von Goldchlorid hergestellt wurden, mit äthylalkoholischen Lösungen von i-Amylalkohol, Benzol und Tetrachlorkohienstofî zu flocken. Auch hierbei konnte besonders schön gezeigt werden, dab die Nichtleiter, allein zum Sol zu. geseizt keine Flockung hervorzurufen vermögen. Bemerkenswert war das Verhalten eines mit Natriumsulfat in biau umgeschlagenen Goldsoles. Dieses schied sich bereits auf Zusaiz der Nichtleiter selbst aus!i).

Ebenso gelang es mir, ein einprozentiges Kieselsäuresol (v. Heyden) zu flocken sowie ein aus Eisenchlorid und Natronlauge nach F. Pow is hergestelltes elektroneganiv geladenes Sol von Eisenhydroxyd, wie auch ein durch Einleiten von Schwefelwasserstoff in Quecksilher. chiorid erhaltenes Quecksilbersulfidsal.

Versuche mit elektropositiv geladenem Sol von Eisenhydroxyd und Aluminium hydroxyd.

Die nach Graham's Methode hergestellten Sole (Eisenhydroxyd 0,55 prozentig und Aluminiumhydroxyd einprozentig) verdankt Herr Professor Traube der Firma Heyden.

Es gelang nicht, im Gegensatz zu den elektronegativgeladenen Solen, diese elektropositiv geladenen so zu flocken.

11) Siehe auch Zsigmondy, Kolloidchemie 2. Aufl (Leipzig 1918), 136.
Theoretische Berrachangen.

Aus obigen Versuchen gehen det iur die theortische Auswertung duse Vo tige Tutsachen iteryor: ersens, lab fie tocknty

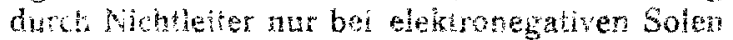
geirnt, bei den posiven dagogea nicht; 2 wei. tens, daß die Flockung wui dann erfolgt, wenn der Sättigungspunkt der Lösung an flockungs" milel uberschitten ist und dritens, dab dieselbe nur durch alkoholische Lösungen dieser Substanzen bewirk wirkt. Setzi man die Nichtleiter allein an den Kolloiden, so eriolgt auch bei heftigstem Schürteln keine oder nur eine geringe Flockung. Dagegen sind bei Suspensionen und auch beim blauen Goldsol - also auch bei gröber dispersen Systemen - die Nichtleiter selbst wirksam.

Auf Grund dieser Tatsachen kann folgende Erklärung gegeben werden:

Die Nichteiektrolytflockung der hochdispersen Kolloide erfolgt in zwei Phasen. Die erste Phase besteht aus einer Dispersitätsgradverminderung una die zweite aus einer Adsorption der nunmehr crtaltenen größeren Teilchen an den Grenzlächen der ausgeschiedenen Kügelchen des Fillungsmittels.

Aus der vorhergehenden Arbeit von $\mathrm{Tr} a \mathrm{ub} e$ und $\mathrm{Kle}$ in geht nun hervor, daB alle die hier angewandten Lösungen von oberflächenaktiven Stoffen, wie selbst von i-Amylalkohol, Anilin usw, in der Nâhe ihres Sättigungspunktes eine erhebliche Anzahl von Subnikronen enthaiten. Wird nun der Sättigungspunkt überschritten, indem dem Hydrosol eine grobe Menge des Flockungsmittels in alkoholischer Lösung zugesetzt wird, so hinterbleibt dasselbe nach der Aullosing des Alkohols in Wasser in eine: hochdispersen, jedoch recht labilen Form. In diesem Zeirpunkt besteht demnach ein sehr unbeständiges Gemisch des zu flockenden Kolloils und des hochdispersen Flockungsmittels. Es liegt daher nahe, die genannte erste $\mathrm{Ph}$ a se auf eine gegenseitige flockung $z$ weier entgegengesetzt geladener Kolloide zurückzuführen.

Diese Auffassungsweise enthält eine Schwie. rigkeit. Sie erklärt nur die Flockung durch Nichtleiter, nicht die phänomenologisch ebensc erfolgende Flockung durch schwer lösliche ober. tâchenaktive Säuren und Basen; deren kolloide Lösungen haben nämlich, wie aus der erwähnten Arbeit von $T$ raube und Klein hervorgeht, negative Ladung. Diese Schwierigkeit kann vielleicht vermieden werden, wenn wir bedenken, daß die gesättigten Lösungen der ge- 
narnten Säuren und Basen nur zum Teil kolloid sind, tellweise sind sie jedoch molekulardispers und dementsprechend auch ionisiert. Wir hätten also in diesen Pällen als erste Phase statt mit einer gegenseitigen Flockung yon Kolloiden mit einer gewöhnlichen Elektrolyt. flockung zu tur.

\section{Zus a men $\mathrm{f}$ as ung.}

1. Oberflächenaktive Nichtleiter sind auch ohne Mitwirkung von Elektrolyten befähigt, Plockungen von Suspensionen wie negativ geladenen Kolloiden (Suspensoiden und Emulsoiden) uerbeizuführen. Nach früheren Versuchen von Traube werden Suspensionen von Kohle, Schwefel usw. durch oberfiächenaktive Stotfe geflockt, sobald die wässerige Lösung an. diesen Stoffe gesähtigt ist. Für Sole elektronegativ geladener Kolloide, wie Arsensulfid, Eiweib, Gold, Kieselsäure und Quecksibersulfid gilt dasselbe. Die Flockung erfolgt indessen nur dann, wenn der oberflachenaktive Stoff itn Zustande feinster Verteilung zweckmätig in Porm athylalkoholischer Lösung z"* gefügt wird. Elektropositiv geladene Sole, wie Eisenoxydhydrat und Aluminiumoxydhydrat werdest nicht geflockt.

2. Die Flockung einer Eiweiblosung durch Narkotika ist zum mindesten solange irreversibel, als das Narkotkum am EiweiB adsorbiert ist. Diese Tatsache dürfte für die schädlichen und tödichen Wirkungen der Narkotika bedeutungsroll sein.

3. Die Flockung durch beschränkt lösliche oberflächenaktive Nichtieiter erfoigt vermutlich in zwei Phasen. Die erste Phase besteht aus einer Dispersitätsgradverminderung durch gegenseitige Flockung zweier Kolloide: die zweite aus der Adsorption der nunmehr gevildeten größeren Teilchen an den Grenzfläcien der ausgeschiedenen Tröpfchen des Nichtleiters. Diese Auffassung erfordert eine positive Ladung für die primär gebildeten Kolloide der Nichtleiter.

Anmerkung von J. Traube. Ich halte die Hypothese von Herrn Klein für sehr beachtenswert. Vielleicht darf ich aber meinerseits noch darauf aufmerksam machen, da6 für oberflächenaktive Stoffe, wie beispielsweise i-Amylalkohol, die Oberflächenspannung der gesättigten wässerigen Lösung gegen Luft sehr angenähert gleich ist der Oberflächenspannung des i-Amylalkohols als solchem. Angenommen nun, es handle sich um eine Koh̀lesuspension, so wird an der Oberfläche der Kohle eine Schicht Amylalkohols oder eine hochprozentige wässerige Lösung desselben adsorbiert. Solange nun die Kohle in einer verdinnten $\mathbf{i}$-Amylalkohollösung suspendiert ist, besteht cine Grenzflächenspannung zwischen der adsorbierten Schicht und dem Dispersionsmittel. Diese Grenzflächensparnung wird aber gleich oder nahezu gleich 0 im Falle der Sättigung der Lösung. In diesem Augenblick erfolgt die Flockung. Sollte nicht ein Zusammenhang bestehen können zwischen jenen beiden Faktoren? Man hätte dann anzunehmen, daB durch den Wegfall der Grenzflächenspannung die die Kohleteilchen trennende Kraft verschwindet, und es wäre eine gewisse Analogie hergestellt $z u$ den Vorgängen in der isoelektrischen Zone, nur dab hier an Stelle der elektrischen Ladungen die Oberfiächenspannungen zu setzen wären.

Ich möchte hier nur das Problem von einem anderen hypothetischen Standpunkte aus beleuchten, ohne gegen die mir recht sympathische Hypothese des Herrn Kiein Stellung zu nehmen.

\section{Ein Beitrag zur Katalyse des Wasserstoffperoxydes durch kolloides $\mathrm{MnO}_{2}$.}

Von A. Lottermoser und R. Lehmann. (Bingegangon 12. Auguat 1921.)

Gelegentlich eines Gutachtens über Sauerstoffbäder hat der eine von uns (Lottermoser) eine starke Abhängigkeit des Verlautes der Wasserstoffperoxydkatalyse von der Art der in natürlichem Wasser gelösten Elektrolyten benbachtet, so dat der Wunsch entstand, diese Beeinflussung genauer 2 id studieren. Es handelte sich bei dem erwähnten Gutachten um einen Zusatz von Perborat und Kaliumpermanganat zum Badewasser in solchen Mengen, dal in der Badezeit von ca. 30 Minuten aller Sauerstoff in Freiheit gesetzt wurde. Derselbe bleibt aber it übersättigter Lösung und entwickelt sich an der Haut des Badenden in 